У дерева есть корневой узел и конечные узлы. После создания корневого узла мы можем построить дерево, выполнив две части:

1. При создании терминальных узлов дерева решений одним из важных моментов является решение о том, когда прекратить выращивание дерева или создать дополнительные терминальные узлы. Это может быть сделано с использованием двух критериев, а именно: максимальная глубина дерева и минимальная запись узла следующим образом:

- Максимальная глубина дерева — как следует из названия, это максимальное количество узлов в дереве после корневого узла. Мы должны прекратить добавлять терминальные узлы, как только дерево достигнет максимальной глубины, т.е. как только дерево получит максимальное количество терминальных узлов.

- Записи минимального узла- его можно определить как минимальное количество шаблонов обучения, за которое отвечает данный узел. Мы должны прекратить добавлять терминальные узлы, когда дерево достигнет этих минимальных записей узлов или ниже этого минимума.

2. Терминальный узел используется для окончательного прогноза.

Рекурсивное расщепление - это метод построения дерева. В этом методе после создания узла мы можем рекурсивно создавать дочерние узлы (узлы, добавленные к существующему узлу) для каждой группы данных, сгенерированной путем разделения набора данных, вызывая ту же функцию снова и снова.

После построения дерева решений нам нужно сделать прогноз об этом. По сути, прогнозирование предполагает навигацию по дереву решений с помощью специально предоставленной строки данных.

Мы можем сделать прогноз с помощью рекурсивной функции, как это было сделано выше. Та же самая процедура предсказания вызывается снова с левыми или дочерними правыми узлами.

$* * *$

1. Уэс Маккинли Python и анализ данных / Пер. с англ. Слинкин А.А. - М.: ДМК Пресс, 2015, -482 c: ил.

2. Рашка C.Python и машинное обучение / пер. с англ. А.В. Логунова. - М.: ДМК Пресс, 2017. - 418 с.: ил.

3. «Открытый курс машинного обучения: анализ временных рядов с помощью Руthon [Электронный pecypc] Режим доступа: Интернет: https://habr.com/ru/company/ods/blog/327242

4. Обзор методов классификации в машинном обучении [Электронный ресурс] Режим доступа: Интернет: https://tproger.ru/translations/scikit- learn-in-python

\title{
Люкин Д.В. \\ Различные подходы к решению проблемы рекомендации мультимедийного контента
}

МИРЭА - Российский технологический университет (Россия, Москва)

doi: $10.18411 / \mathrm{j}-05-2021-16$

\section{Аннотация}

Мультимедийный контент достаточно плотно вошел в жизни людей. Новостные ресурсы все чаще прилагают различные изображения, аудио и видео сообщения к своим статьям, обучающие ресурсы постепенно переходят с обучения по текстовым материалам на гибридные подходы, владельцы интернет-ресурсов добавляют все больше различных типов медиа на свои сайты, чтобы удовлетворить потребности большего числа пользователей, поэтому сейчас так важно уметь правильно 
рекомендовать такой контент. В этой статье рассмотрены различные подходы к решению проблемы рекомендации мультимедийного контента.

Ключевые слова: мультимедийный контент, рекомендательные системы, на основе контента, коллаборативная фильтрация, нейронные сети, машинное обучение, глубокое обучение, интеграция рекомендательных систем.

\section{Abstract}

Multimedia content has become quite dense in people's lives. News resources are increasingly attaching various images, audio and video messages to their articles, educational resources are gradually moving from teaching based on text materials to hybrid approaches, Internet resource owners are adding more and more different types of media to their sites to meet the needs of a larger number of users. That's why it is so important now to be able to properly recommend such content. This article discusses various approaches to solving the problem of multimedia content recommendation.

Keywords: multimedia content, recommender systems, content-based, collaborative filtering, neural networks, machine learning, deep learning, integration of recommender systems.

\section{Введение}

С постоянно растущим объемом информации в сети рекомендательные системы стали занимать важное место в задаче оптимизации информационного потока как для конкретного пользователя, так и для группы пользователей. Полезность рекомендательных систем нельзя недооценивать, учитывая их широкое распространение во многих веб-приложениях, а также их влияние на решение проблем в области поддержки принятия решений.

Современные системы хранят и обрабатывают более сложные данные, представляющиеся одновременно в разных формах (видео, аудио, текст и т.д.). Такие данные называются мультимедиа, а контент, содержащий в себе эти данные, мультимедийным.

\section{Рекомендательные системы}

Рекомендательные системы - это комплексы алгоритмов, программы и сервисы, основная задача которых предсказать, какие объекты (товары или услуги) будут интересны пользователю, имея информацию о его профиле либо иные данные. Другими словами, рекомендательная система - это система, которая стремится предсказать уровень заинтересованности пользователя к конкретному объекту. Рекомендательные системы используются в различных областях и находят широкое применение в большинстве сегодняшних веб-приложений для рекомендации товаров, новостных статей, медиа и мультимедиа контента.

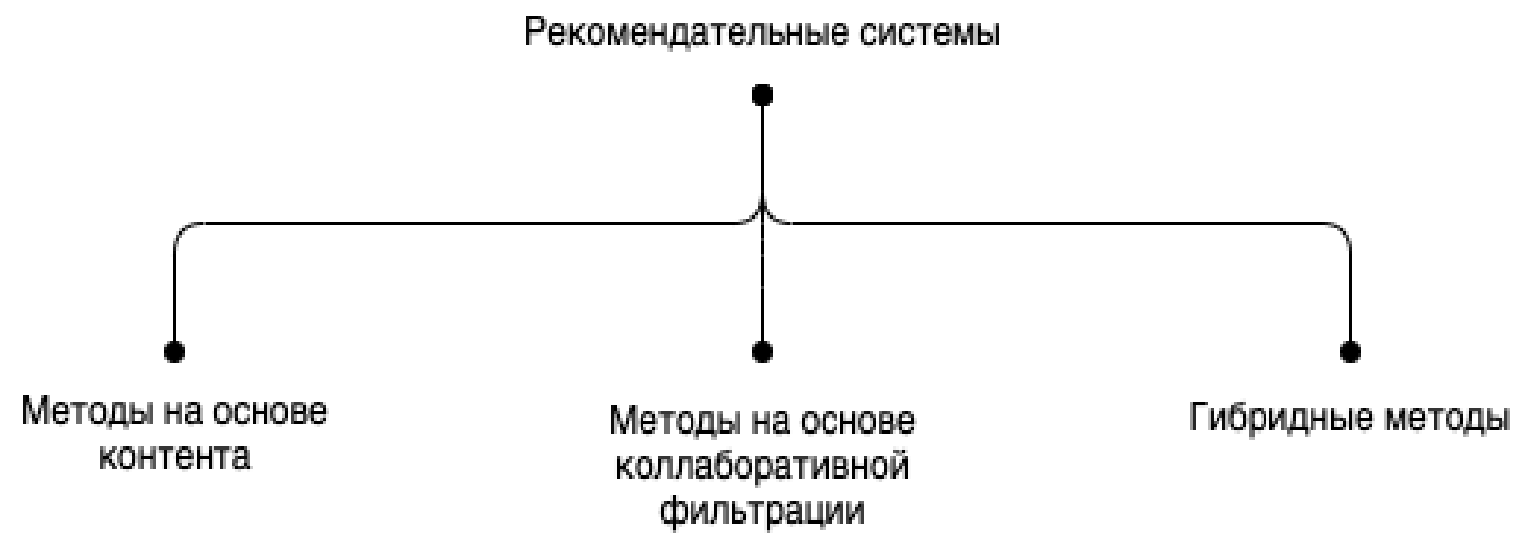

Рисунок 1 - Рекомендательные системы 
Рекомендательные системы, далее РС, обычно подразделяют на три категории (рис. 1): РС на основе алгоритмов коллаборативной фильтрации, РС, на основе контента, и гибридные РС, где рекомендации основаны на комбинировании коллаборативных и контентных подходов.

\section{Рекомендации на основе контента}

Рекомендательные системы на основе контента пытаются предсказать поведение пользователя, основываясь на свойствах объектов, с которыми этот пользователь взаимодействовал (рис. 2).

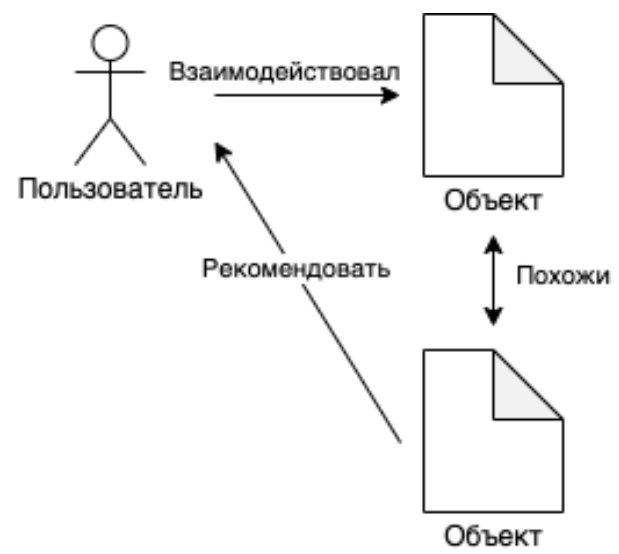

Рисунок 2 - Рекомендаџии на основе контента

Для реализации этого метода в системе помимо самих пользователей и объектов, где объект - это одна единица мультимедийного материала, представленного в системе, необходимо иметь массив свойств. Эти свойства могут отображать степень принадлежности к какой-либо категории, описывать подходящие для каждого отдельного объекта теги или содержать метаинформацию, такую как время создания, язык, автора и т.д. С помощью этих свойств необходимо описать как объекты (степень принадлежности каждого свойства к каждому объекту), так и самих пользователей (степень принадлежности каждого свойства к каждому пользователю).

Эти свойства могут задаваться как самими пользователями, например, создание новых тегов для нового материала или добавление категорий в пользовательский профиль, так и присваиваться каждому пользователю и объекту самой системой исходя из последних действий пользователей.

Одним из таких свойств описывается наличие в объекте той или иной формы данных. Так, например, объект 1 помимо статьи содержит в себе прикрепленные видеоматериалы, которым данный пользователь отдает предпочтение (рис. 3).

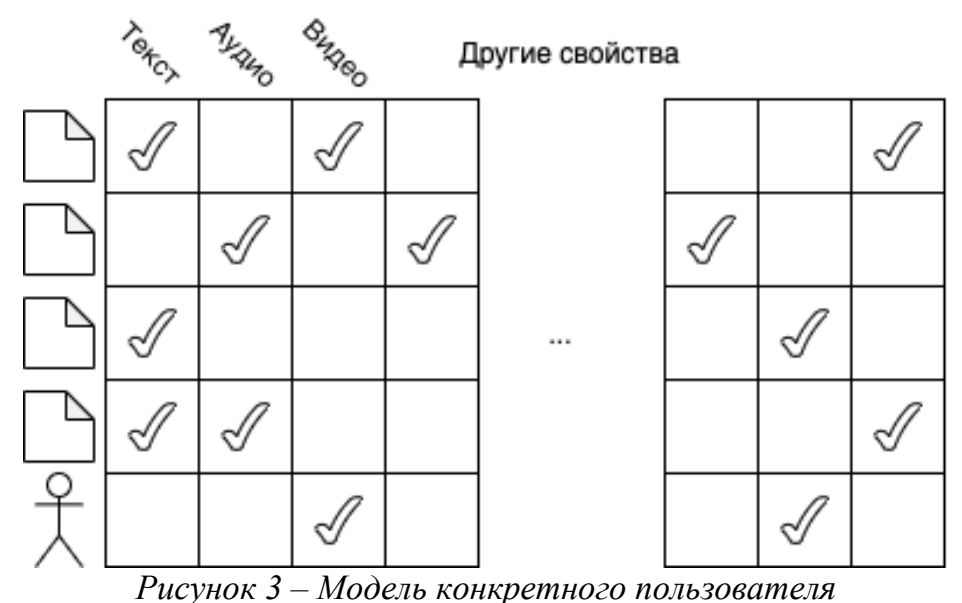

Рисунок 3 - Модель конкретного пользователя 
По всем данным строится модель (каждая такая модель описывает конкретного пользователя и не будет работать для других пользователей). На основе этой модели и составляются рекомендации. Для этого достаточно найти похожие на конкретного пользователя объекты любым известным способом, например, можно перемножить векторы каждого объекта и данного пользователя и выбрать по итогу объекты с самым высоким итоговым результатом (близким к 1).

Методы, основанные на контенте, гораздо меньше страдают от проблемы холодного старта (невозможность дать точные рекомендации для новых пользователей с небольших количеством информации о них), чем другие подходы: новые пользователи и элементы описываются их свойствами, поэтому для этих новых сущностей можно сделать соответствующие предложения.

\section{Системы коллаборативной фильтрации}

Коллаборативные системы описывают каждого отдельного пользователя и объект как вектор признаков, затем отражают их (векторы) в одно пространство. Такие системы учитывают реакцию других пользователей, когда вычисляют рекомендации для конкретного пользователя, выделяют, какие объекты нравятся конкретному пользователю, а также объекты, которые нравятся пользователям с таким же поведением и предпочтениями, собирают отзывы пользователей о различных объектах и используют их для рекомендаций (рис. 4).

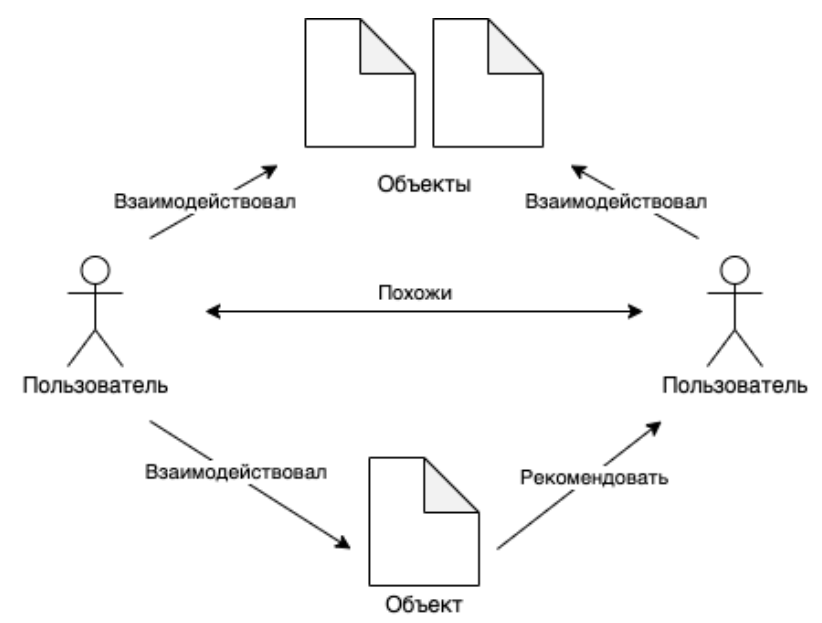

Рисунок 4 - Системы коллаборативной фильтрации

Коллаборативные методы - это методы, основанные исключительно на прошлых взаимодействиях пользователей с объектами. Эти взаимодействия хранятся в так называемой матрице взаимодействий (рис. 5).

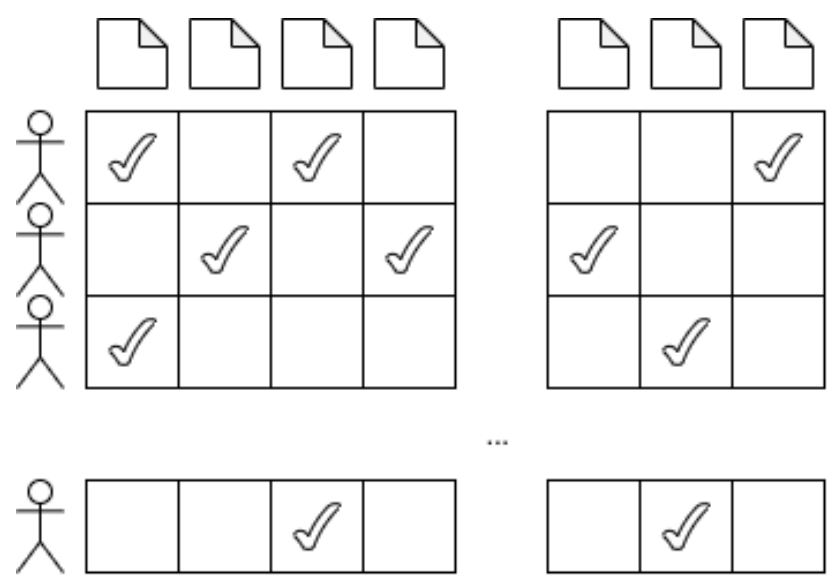

Рисунок 5-Матрица взаимодействий 
Основная идея заключается в том, что взаимодействий между пользователями и объектами достаточно для обнаружения похожих пользователей и/или аналогичных объектов и выполнения прогнозов на основе этих данных.

Для реализации данного метода необходимо взять множество всех пользователей и объектов в системе и отразить в $\mathrm{N}$-мерное пространство. В данном примере используется трехмерное пространство. Каждый элемент выходного вектора $<\mathrm{x}, \mathrm{y}, \mathrm{z}>$ будет соответствовать степени присутствия в каждом отдельно взятом объекте текстового, аудио и видео контентов соответственно. Для пользователя же это будет означать степень заинтересованности пользователя в каждом из этих видов контента. Тогда матрица взаимодействия для какого-то случайного множества пользователей и материалов будет выглядеть следующим образом:

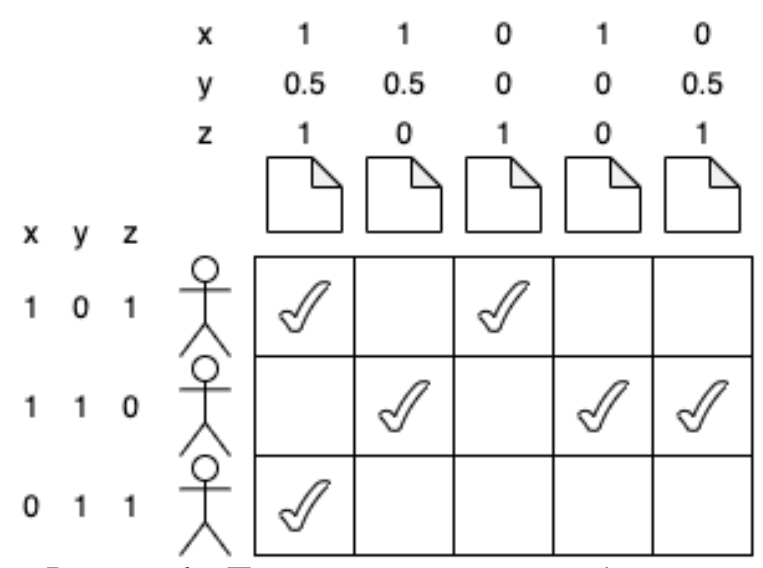

Рисунок 6-Пример матрищы взаимодествия

И соответственно графическое представление этого отражения будет выглядеть так:

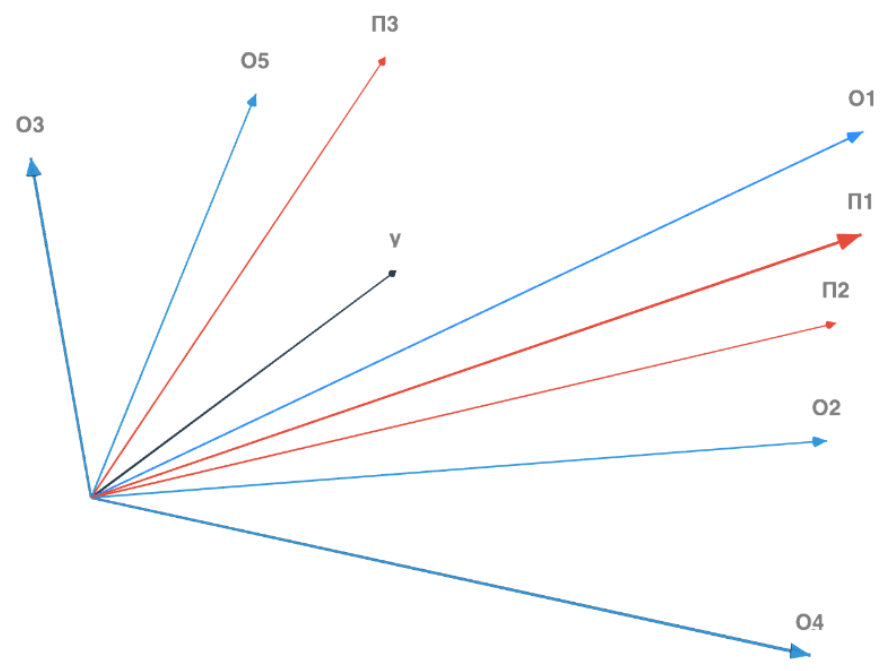

Рисунок 7 - Графическое представление матрищы взаимодействий

Здесь соседние элементы наиболее похожи (или, если говорить о взаимоотношении пользователь-объект, наиболее подходят друг другу). Таким образом, имея обученную модель, можно для конкретного пользователя найти похожих на него пользователей и рекомендовать просмотренные ими материалы.

В качестве примера были приведены конкретные свойства, которыми описывались взаимодействия пользователь-объект, но эти свойства могут быть сгенерированы (получены автоматически). В этом и заключается вся мощь методов 
коллаборативной фильтрации. Метод генерации таких свойств называется матричной факторизацией.

В основе матричной факторизации лежит предположение, что существует пространство свойств с небольшой размерностью, в котором можно представить как пользователей, так и объекты, такое, что взаимодействие между пользователем и объектом может быть получено путем вычисления произведения соответствующих векторов в этом пространстве.

Однако свойства не подаются в модель напрямую. Система сама обнаруживает эти полезные свойства. Они (свойства), взятые по отдельности, имеют математическое значение, но не имеют интуитивной интерпретации. Однако структуры, возникающие из этого типа алгоритмов, очень близки к интуитивному разложению. Следствием такой факторизации является то, что близкие пользователи с точки зрения предпочтений, а также близкие элементы с точки зрения характеристик в итоге имеют близкие представления в скрытом пространстве.

Существует несколько алгоритмов матричной факторизации, но в данном случае будет использоваться Funk SVD, разработанный Саймоном Фанком для решения проблем классического SVD.

Входными данными будет являться матрица взаимодействия пользовательобъект и размерность пространства, в которое необходимо отразить модель. Сначала строятся матрицы пользователей и объектов по количеству свойств. Матрицы заполняются случайными значениями. Далее находится скалярное произведение для конкретного отношения пользователь-объект и сравнивается со значением в исходной матрице. Для измерения качества рекомендаций берется среднеквадратичная ошибка. На следующем шаге необходимо минимизировать ошибку, используя градиентный спуск, обновить значения, вычислить скалярное произведение и вычислить ошибку для нового прогноза. Через какие-то количество итераций модель обучится и будет выдавать достаточно хорошие результаты.

Для учета пользовательских предпочтений относительно типа контента в объекте можно добавить собственные свойства, но это не обязательно, так как если какой-то пользователь явно предрасположен к какому-то определенному типу контента, система сможет это понять (благодаря вышеописанному алгоритму) и составит соответствующую модель.

Основное преимущество коллаборативных подходов состоит в том, что они не требуют информации о пользователях или объектах, поэтому их можно использовать во многих ситуациях. Более того, чем больше пользователей взаимодействуют с объектами, тем более точными становятся новые рекомендации. Однако, поскольку для выработки рекомендаций учитываются только прошлые взаимодействия, коллаборативная фильтрация страдает от проблемы холодного старта.

\section{Рекомендательные системы с применением глубокого обучения}

В последнее время глубокие нейронные сети кардинально меняют архитектуру рекомендаций и предоставляют больше возможностей для повышения производительности таких систем. Последние достижения в системах рекомендаций, основанных на глубоком обучении, получили значительное внимание, решая проблемы традиционных моделей и достигая высокого качества рекомендаций.

В области рекомендаций в последнее время появилось множество различных интеллектуальных решений: модели на основе персептронов и автоэнкодеров, рекомендации на основе сессии, глубокое векторное представление и другие. Для решения проблемы рекомендации мультимедийного контента лучше всего подходит метод нейронной коллаборативной фильтрации.

Модель берет два отдельных вектора, один из которых отражает пользователя, второй - объекты. Вектор объектов имеет единицы по всем индексам, с которым пользователь взаимодействовал (рис. 8). 


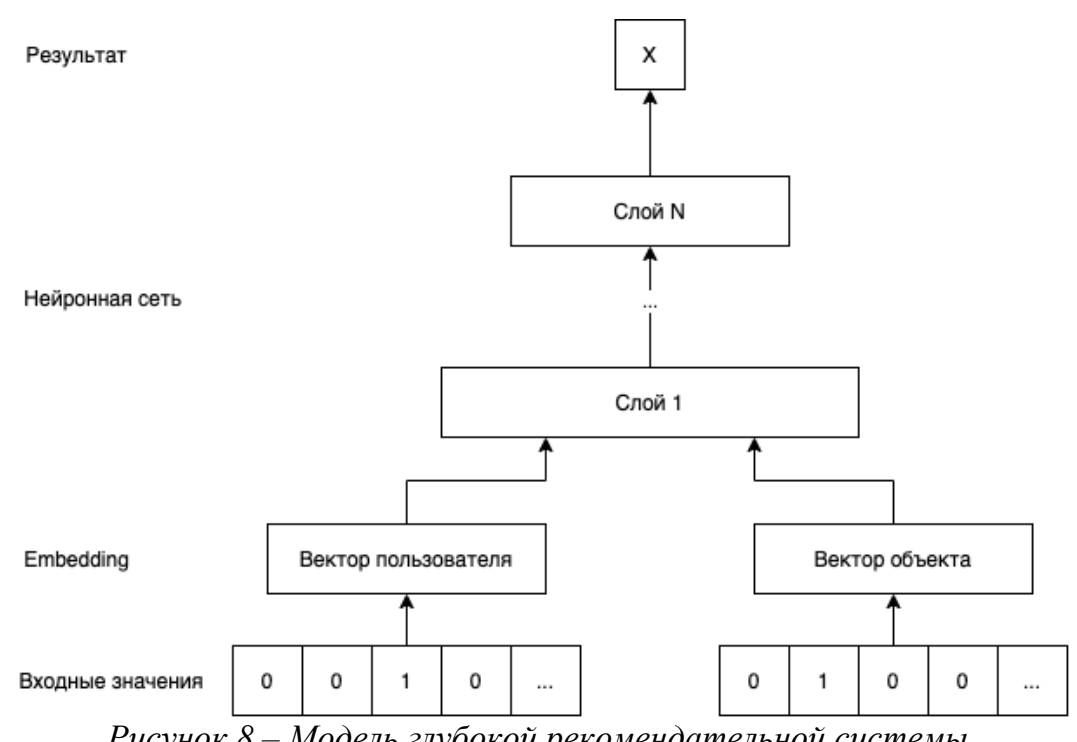

Следующие слои преобразуют входные векторы в отраженные в пространство свойств скрытые векторы, которые далее подаются в многослойную нейронную сеть для преобразования в прогнозируемые оценки вероятности. Слои отвечают за поиск сложных отношений между пользователем и объектом в входных данных. Выходной слой выдает прогнозируемую оценку того, насколько велика вероятность того, что пользователь будет взаимодействовать с объектом.

\section{Гибридные подходы}

Гибридные подходы используют информацию как о взаимодействиях пользователей с объектами, так и об их характеристиках. В сочетании с моделями глубокого обучения они могут быть достаточно эффективными. Так, например, рекомендательная система YouTube, основанная на модели глубокого обучения, для прогнозирования просмотра пользователями учитывает их предыдущие действия (поисковые запросы и просмотренные видео) и статическую информацию (пол, местоположение и т. д.). Просмотренные видео и запросы представлены в виде векторов. Поскольку нейронные сети обычно принимают входные данные фиксированной длины, векторы видео или запросов, просмотренные пользователем, усредняются и объединяются с другими статическими функциями.

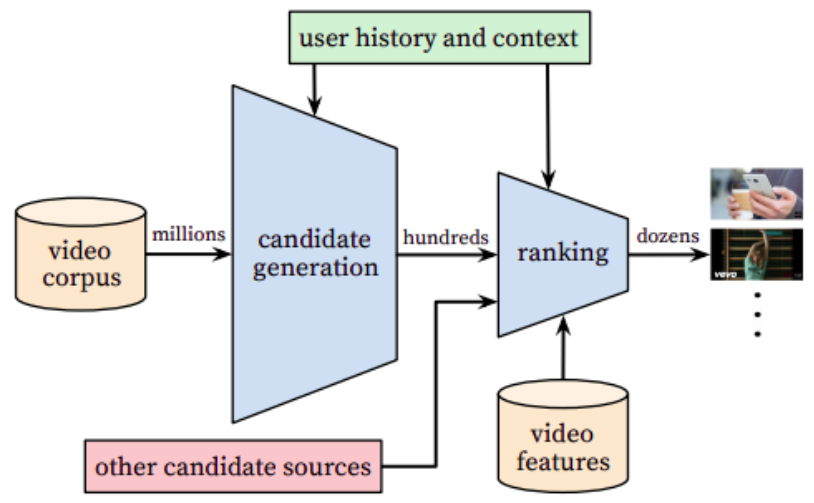

Рисунок 9-Система рекомендаций YоиТиbе [3]

Система состоит из двух нейронных систем (рис. 9). Первая - это нейронная сеть генерации кандидатов, вторая - ранжирования. Сеть генерации кандидатов берет события из истории пользователей YouTube и персонализирует их с помощью коллаборативной фильтрации. Затем пользователи сравниваются по различным 
свойствам, как, например, количество просмотренных видео, демографическая информация и токены поисковых запросов. Несколько иначе работает сеть ранжирования. Она присваивает оценку каждому видео, используя богатый набор свойств, описывающих видео и пользователя. Эта двухуровневая система позволяет обрабатывать миллионы видео, а также масштабировать ее до отдельных пользователей и предоставлять им значимый контент.
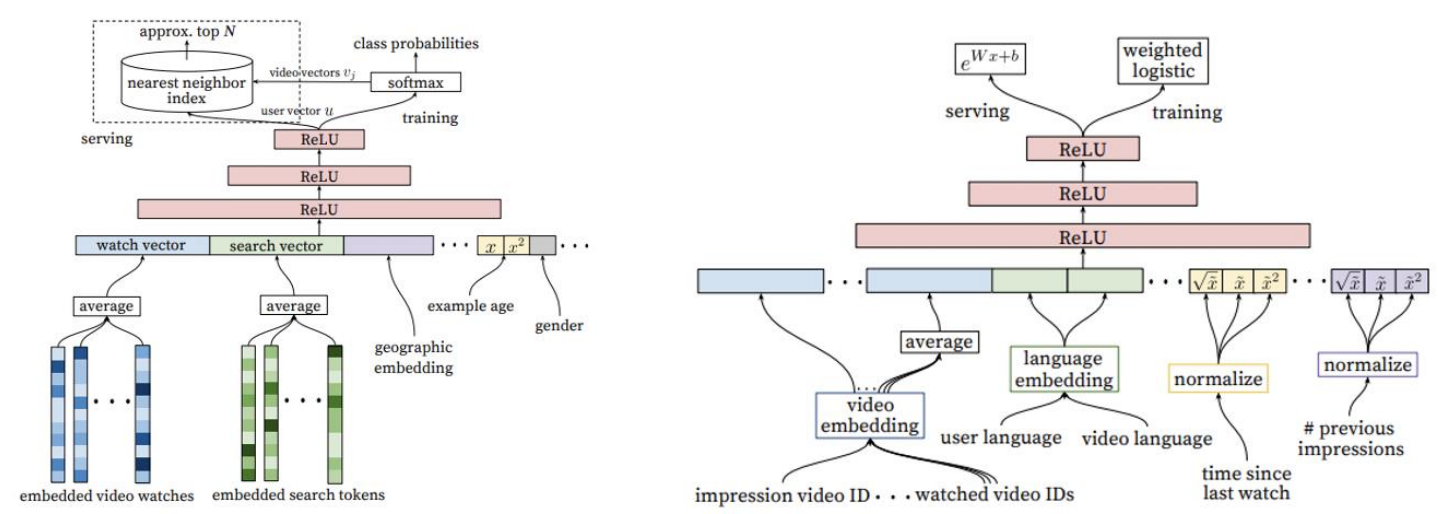

Рисунок 10 - Модели генерации кандидатов и ранжирования ҮоиТиве [3]

Обе системы на входе имеют embedding слои, катинирующие свои выходы в один общий слой, подаваемый на вход группе скрытых слоев с функцией активации ReLU. Отличие лишь в задаче, которую они решают. Таким образом генератор кандидатов ищет наиболее похожие объекты, а сеть ранжирования в свою очередь на выход отдает приблизительную оценку, которую пользователь может поставить объекту, и в дальнейшем сортирует всех отобранных кандидатов по этой оценке.

Гибридные методы используют алгоритмы рекомендаций, основанных на контенте, когда пользователи и объекты неактивны или малоактивны, и становятся более точными по мере появления большего количества данных за счет использования коллаборативной фильтрации.

\section{Интеграция рекомендаций в целевую систему}

Сервис рекомендаций лучше всего реализовывать как отдельный микросервис, при этом стоит ограничить ему доступ к базе данных, то есть все входные данные как для обучения, так и для рекомендации он будет получать из основного сервиса (рис. $11)$.

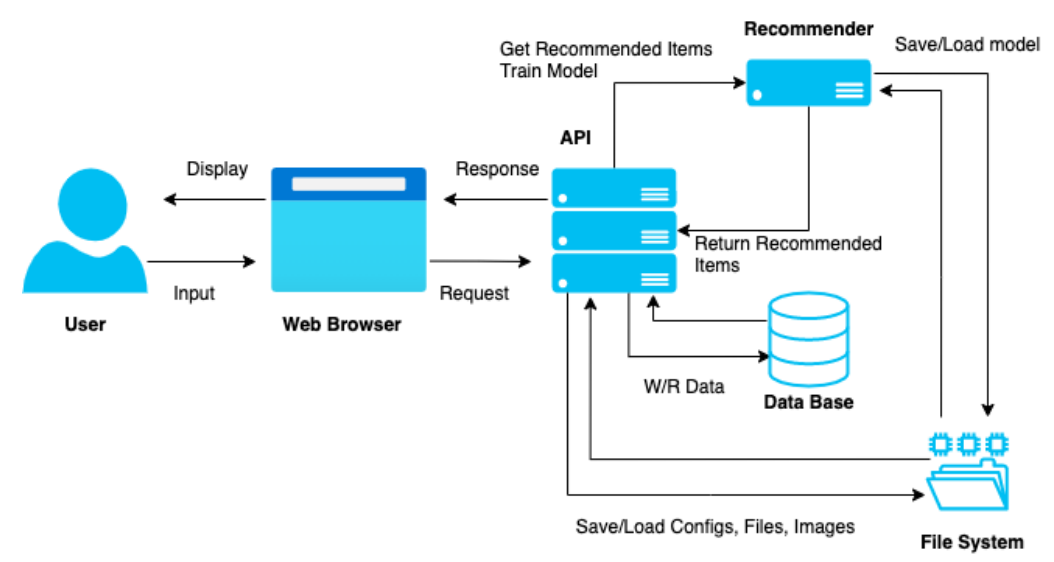

Рисунок 11 - Пример архитектуры системы с рекомендациями

Когда сервер получает запрос на генерацию рекомендаций для конкретного пользователя, он в зависимости от способа реализации будет: 
- искать конкретного пользователя в матрице (для матричной факторизации и контентного подхода);

- вычислять описание пользователя через отражение в пространство заданных свойств в рантайме (для подхода глубоких рекомендаций).

Когда описание пользователя (или объекта) найдено, система возвращает $\mathrm{K}$ ближайших соседей из пространства свойств. Полученные объекты и будут являться рекомендацией для пользователя.

Далее можно пропустить полученные рекомендации через систему оценки, чтобы отсортировать их по релевантности. Это точно такая же рекомендательная модель, только нацелена она на предсказание оценки пользователя конкретного материала.

\section{Вывод}

В данной статье были рассмотрены основные подходы к решению проблемы рекомендации мультимедийного контента, представлены способы их реализации, продемонстрированы преимущества и недостатки каждого из них, описаны способы интеграции рекомендаций в целевую систему.

$$
* * *
$$

1. James Le, Alexander G. Ororbi. From Matrix Factorization To Deep Neural Networks: The Evolution Of Collaborative Filtering Solutions for Recommendation Systems. Rochester Institute of Technology. 2018 -10 страниц.

2. Shuai Zhang, Lina Yao, Aixin Sun и Yi Tay. Deep Learning based Recommender System: A Survey and New Perspectives. ACM Comput. Surv. 2018 - 35 страниц.

3. Paul Covington, Jay Adams, Emre Sargin. Deep Neural Networks for YouTube Recommendations. RecSys. 2016 - 8 страниц.

4. Сорокин А.Б. Искусственные нейронные сети прямого распространения: учебно-методическое пособие / Сорокин А.Б., О.В. Платонова. - М.: МИРЭА - Российский технологический университет, 2018. - 68c

5. Recommendations Systems [Электронный pecypc] URL: https://developers.google.com/machinelearning/recommendation (дата обращения: 15.04.2021)

\section{Напрасникова К.А., Перова М.В. \\ ЭДО: мифы и реалии электронного документооборота}

Южно-Российский институт управления - филиал Российской академии народного хозяйства и государственной службы при Президенте Российской Федераџии

doi: $10.18411 / \mathrm{j}-05-2021-17$

(Россия, Ростов-на-Дону)

\section{Аннотация}

В статье описаны существующие вокруг электронного документооборота мифы, обоснование их реальности, а также определение удобства работы с бумагой или «цифрой».

Ключевые слова: электронный документооборот, миф, реальность, бизнес, безопасность, информация.

\section{Abstract}

The article describes the myths that exist around Electronic document flow (EDF), the rationale for their reality, as well as the definition of the convenience of working with paper or "digital". information.

Keywords: electronic document flow (EDF), myth, reality, business, security,

В современном мире с учетом быстро протекающих процессов цифровизации многие компании, особенно имеющие филиалы, переходят на электронный 